\title{
The Raman Effect and Chemical Structure
}

IN the May number of Scientia, Prof. K. W. F. Kohlrausch discusses at some length the significance of the so-called Raman optical effect. The outstanding importance of this phenomenon, which had been foreshadowed by Smekal from theoretical considerations some four and a half years before its actual discovery early in 1928 by Raman, is evident from the unusually large number of publications which have appeared upon the subject.

Experiment has shown that, whereas the valency electrons are stimulated only by high frequency waves, inter-atomic vibrations can be produced by lowfrequency radiation; and of the various ways in which such inter-atomic vibrations can be excited, that due to Raman is by far the easiest to carry out experimentally. The apparatus required is the ordinary spectrophotographic outfit. The substances to be investigated, preferably colourless solids or liquids, are intensely illuminated with discontinuous light such as that emitted by a quartz mercury vapour lamp, and the horizontally scattered light is then photographed spectrographically ; when, in addition to the usual characteristic lines of the spectrum, weaker satellites are found, the distances of which from the standard lines are a measure of the frequencies of the inter-atomic vibrations. Now, if reasonable assumptions can be made about the forces which are involved, it is clear that most valuable deductions may be drawn about the molecular structure.

It must be remembered, however, that the method has serious limitations, for in many cases the intensity of the lines becomes vanishingly small. Thus comparison of the results with those obtained by the older method of measuring infra-red absorption bands should be carried out wherever possible as controls, since the two methods of investigation are complementary.

The investigation of molecular structure is by no means simple, although some progress has been made. The simplest case is that of the diatomic molecule, since only one kind of vibration is possible and the
Raman spectrum consists of a single line, apart from any lines due to rotation of the molecule and having frequencies of a different order of magnitude. Further, it is recognised that certain uni- or multivalent radicals can be regarded as single atoms for the purpose of calculation. These radicals possess characteristic "inner" vibrations, which are independent of the "outer' vibrations of whole radicals within the molecule. Such 'inner' frequencies are in fact constitutive factors and can be determined quantitatively. Thus it is possible to calculate the value of the "mean restoring force' $K$ between two carbon atoms which are united by single, double, or triple bonds. Since the values obtained for $K$ are approximately in the ratio $1: 2: 3$, it appears that these old-fashioned valency 'bonds' have some mathematical justification, although it is necessary to bear in mind that other factors might influence the attractive force between the atoms. Molecules consisting of more than two atoms or radicals are more difficult to deal with, but even here some progress has been made by grouping some of the atoms into radicals, and also by taking advantage of the fact that the spatial arrangement can often be determined by purely chemical methods.

The usefulness of the method may be illustrated by its application to the much-disputed case of nitric acid and of other isodynamic molecules, such as prussic acid, acetoacetic ester, etc. In the case of nitric acid, the evidence points to the fact that in the anhydrous condition the structure is exclusively $\mathrm{HO} . \mathrm{NO}_{2}$, whereas on the addition of water, some of the molecules undergo change, since the characteristic spectrum of the anion $\overline{N O}_{3}$ can be detected. Thus the number, position, intensity, and polarisation of the lines in the Raman spectra are determined not only by the spatial arrangement of the atoms within the molecule, but also by the attractive forces between them, and the complete interpretation of these spectra will facilitate a quantitative knowledge of molecular structure, such as has never before been possible.

\section{Association of Teachers in Technical Institutions}

\section{Annual Conference}

" WE can leave to those who seek them the some. what Pyrrhic victories over ignorance of a purely academic education with its exaggerated claims to culture, whilst the general body of mankind proceeds to enjoy the conquests won by technical efficiency in producing the necessities of life. ..." That might be said to be the keynote of the first part of the presidential address delivered by Mr. S. H. Moorfield to the Annual Conference of the Association of Teachers in Technical Institutions held in Cardiff during Whitsuntide. Clearly and forcibly he urged the claims of technical education to a foremost position in the educational systems of mankind in what is obviously a rapidly changing world.

In spite of the fact that Hero described the steam turbine about 200 B.c., that Archimedes was credited with the invention of the screw, that Torricelli taught us much of the mechanics of fluids, that Francis Bacon experimented upon the preservation of food by freezing, and that James Watt definitely applied scientific principles to the improvement of the steam engine, technical education as we know it is but some eighty years old. During that period, human achieve- ment has been amazing. Transport has undergone transformation which may be well called revolutionary. Electricity has been brought to the service of every department of life, and has brought speed, cleanliness, and convenience in its train. Fifty years ago there was no electric motor in industry. Wireless telegraphy and telephony in their present form and with their world-wide range were undreamt of ; electric lighting and heating were nothing more than laboratory experiments. Experts in every sphere of life may now catalogue long lists of conquests. In the presence of these vast changes, it is illogical to maintain that the education of mankind should follow the same course that it has followed for centuries. New environment needs a fresh adaptation of the equipment which enables life to be efficiently sustained.

All this is true. Yet, while lip service is paid to the need for a change in educational policy, there remains much to be done "to combat the influence of reactionaries" who still wield so great an influence. "We find them in posts of educational administration, usually products of the older universities, still living 
with their feet upon the solid earth of the twentieth century, but with their heads in the mists of the middle ages." As to the old reply that education must be 'liberal', Mr. Moorfield was very clear : "Whilst we do not scorn to provide instruction in matters relating to the manual dexterity of the artizan, we emphasise the necessity for the deeper understanding which is provided in a complete technical course designed to meet the needs of those engaged in particular occupations. Such a course is a continuation of a liberal education and cannot in any sense be narrow. Contacts with other occupations are constantly being made, and the engineer begins to understand the miner and the miner the engineer, and the builder will make the acquaintance of both." "We stand for that kind of education which will enable human beings so completely to understand the forces of Nature that the fear of these things, which, in the past, has bred devil-worship, witchcraft, and superstitions in general, may be banished from their lives and cease to burden them with oppression." Further, technical education is " the very spearhead of the attack upon drudgery and want, the fundamental causes of such a large proportion of human unhappiness ".

Modern civilisation, however, involves something more than the development of engineering and chemistry and all those departments which come so easily under a scientific heading. They all aim at the successful production which technical efficiency can give. But technical efficiency towards production is not enough. If products be not adequately distributed, their value disappears; and it is in distribution that we find our most pressing problems. Upon the edifice of scientific production we have to impose an equally scientific scheme of distribution. At present the latter is chaotic, and the commercial sides of our great technical institutions are developing schemes of education to remedy the defect. The recent crisis brought to the forefront problems which seem to be baffling even to the expert. There are gluts of wheat, rubber, coffee, tea, iron, copper, tin, and even diamonds. Gold lies uselessly hoarded in the coffers of two or three of the great nations of the earth. "With plenty in the world, men suffer privation and want." It was in this connexion that Mr. Moorfield called for closer liaison between commercial teachers and the general body of technical teachers. Already their function has been laid down in no narrow sense by the Committee on Education for Salesmanship : "Salesmanship as we understand it is a prime function of direction and supreme management. . . . It embraces the study of the fundamental principles of commerce . . . it is therefore education for commerce on its creative, organising, and executive sides."

Among the resolutions dealt with by the Conference was one urging the closer co-operation of education and industry by means of the formation of a national co-ordinating committee composed of representatives of industry, commerce, educational administration, and technical teachers. Such a committee would be invaluable as a clearing-house for ideas and proposals made by similarly constituted committees which have been formed to act regionally. Another resolution urged the remission of part of the period of apprentice. ship for pupils who have followed a full-time course in a technical institution, the extension of opportunity for all entrants into industry to become skilled workers, and the provision of part-time day courses for ap. prentices and other adolescent workers.

The Conference was officially welcomed by the Lord Mayor of Cardiff and Councillor J. Hellyer, deputy chairman of the Education Committee. An exhibition of books and apparatus was held in the Cardiff Technical College. At the annual dinner of the Association on Whit Monday, Prof. George Knox, who has done so much for mining education in South Wales, directed attention to the essentials to industrial success. In addition to capital, raw material, and machinery, suitable personnel to advise upon, organise, and manage these essentials is an absolute necessity. How far, he asked pertinently, has the lack of attention to this matter of personnel contributed to the present position of secondary industries in the South Wales area?

\section{White Dwarf Stars}

THE Halley Lecture delivered at the University of Oxford by Prof. E. A. Milne on May 19 was on the subject of the "White Dwarf Stars". He said that the discovery by Halley of the proper motion of some of the fixed stars led to a remarkable succession of researches in pure astronomy, in modern physics, and in cosmogony generally. The proper motion of one of Halley's stars, Sirius, was found by Bessel not to be uniform, but to contain a periodic element of about fifty years. This led him to suggest that Sirius was in reality double, consisting of a pair of stars, one much fainter than the other. In 1862 a faint star, Sirius $B$, was actually seen by Alvan Clark close to the place that had been theoretically assigned to the supposed companion. In 1915, W. S. Adams at Mount Wilson Observatory succeeded in obtaining a photograph of the spectrum of Sirius $B$, which led to the unexpected conclusion that the density of Sirius $B$ was of the order of one ton to the cubic inch. It was shown by Eddington that this surprising density was not physically improbable, and further, that in the light of Einstein's general theory of relativity, the relative displacement of the lines of the spectrum of Sirius $A$ and Sirius $B$ could be estimated. The measurement when actually carried out by Adams in 1925 gave a result so near that of Eddington's estim- ate that the computed small radius and high density of Sirius $B$ may now be accepted with confidence. A few other stars besides Sirius $B$ are known in which low luminosity and abnormal blueness are combined with high density; these are known as 'white dwarfs'. They are all within five parsecs of the sun, but there is no reason to suppose that this is an abnormal region of space. Consideration of the phenomenon of novaoutbursts and the study of the nuclei of planetary nebulæ lead to the conclusion that the list of dense objects can be largely extended. The physical state of matter at these high densities has been elucidated by R. H. Fowler in the light of the researches of Fermi and Dirac. The existence of white dwarf stars shows that it is possible for any gas to exist in either of two states or phases, the 'perfect' or the 'degenerate" phase; the dense state being identified with that of the second phase of a gas. It is suggested that, as foreshadowed by Bessel in regard to Tycho's nova of 1572 , the system of Sirius may owe its origin to the nova phenomenon of the original Sirius; two companions resulting, of which one re-expanded and the other remained dense. Bessel's anticipation of the interest of these phenomena in relation to our knowledge of the physical constitution of the universe has been amply justified by the course of events. 